\title{
Non-neoplastic pathologic findings in nephrectomy specimens; postoperative renal insufficiency and outcomes
}

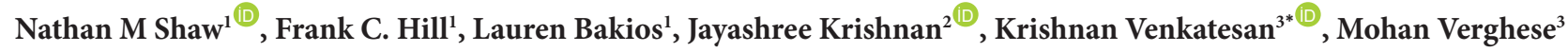 \\ ${ }^{1}$ Department of Urology, MEDSTAR Georgetown University Hospital, Washington, DC, USA \\ ${ }^{2}$ Department of Pathology, MEDSTAR Washington Hospital Center, Washington, DC, USA \\ ${ }^{3}$ Department of Urology, MEDSTAR Washington Hospital Center, Washington, DC, USA
}

\section{A R T I C LE I N F O}

Article Type:

Original

Article History:

Received: 26 September 2019

Accepted: 7 November 2019

ePublished: 27 November 2019

\section{Keywords:}

Renal neoplasm

Renal insufficiency

Nephrectomy

Partial nephrectomy

Pathology

Chronic kidney disease

\begin{abstract}
A B S T RA C T
Introduction: Incidence of renal masses has increased with increased abdominal imagings. The trend in treatment of renal masses has been toward renal preserving options, including surveillance, ablation and partial nephrectomy.

Objectives: To determine the frequency of medical renal disease in patients undergoing surgical intervention for renal neoplasms and to establish whether these pathologic changes predict development of renal insufficiency in the immediate postoperative period.

Patients and Methods: This was an Institutional Review Board (IRB)-approved retrospective review of all patients that underwent radical nephrectomy (RN), partial nephrectomy and nephroureterectomy from December 2009 to November 2013. Around 225 patients had complete pathologic and perioperative data for analysis. We compared preoperative and postoperative glomerular filtration rate (GFR), neoplastic findings, tumor characteristics (positive margins and extracapsular extension), and pathology information regarding non- neoplastic findings (tubular atrophy, chronic inflammation and fibrosis).

Results: The presence of any pathologic abnormalities in the non-neoplastic renal parenchyma was significantly associated with increased serum creatinine levels postoperatively $(P=0.01)$ and at last follow up visit $(P=0.04)$. Univariate analysis showed that glomerular and vascular abnormalities were each significantly associated with worsening renal function.

Conclusion: Our research suggests that abnormalities in non-neoplastic renal parenchyma found in renal specimens after RN should not be ignored as they may predict possible worse outcomes in renal function. This may help make a case for biopsy pre-operatively and a stronger case for nephron sparing surgery. This may also help determine which patients should be followed more closely postoperatively.
\end{abstract}

Implication for health policy/practice/research/medical education:

This work provides further validation to the American Urological Association recommendations regarding testing of nephrectomy specimens for medical renal disease. Additionally, our data suggest screening as early as the post-operative setting is warranted for patients to identify those with renal injury.

Please cite this paper as: Shaw NM, Hill FC, Bakios L, Krishnan J, Venkatesan K, Verghese M. Non-neoplastic pathologic findings in nephrectomy specimens; postoperative renal insufficiency and outcomes. J Renal Inj Prev. 2020; 9(1): e01. doi: 10.15171/jrip.2020.01.

\section{Introduction}

Renal malignancy accounts for approximately $3.8 \%$ of newly diagnosed cancers in the United States according to Surveillance, Epidemiology, and End Results (SEER) database (1). With improvements in abdominal imaging and increased use of these modalities, the incidence of renal masses has increased steadily. The result has been diagnosis of renal masses that are smaller in size and identification of masses in younger patients. Patients now have several options for management of their renal masses including watchful waiting, radical nephrectomy (RN), and nephron-sparing surgery (NSS) (2). As oncologic outcomes continue to improve, more attention has been placed on ways to ensure patients maintain optimal renal function after surgical intervention. This has led to improved surgical technique in NSS and is reflected in the American Urological Association (AUA) guidelines published in 2017 which encourage NSS in cTla renal 
masses (2,3).

There is known and mounting evidence that patients undergoing both RN and NSS have varying degrees of previously undiagnosed underlying medical renal disease that may place patients at greater risk of developing chronic kidney disease (CKD) postoperatively. Salvatore et al found that approximately $15 \%$ of nephrectomy specimens had findings in the non-neoplastic parenchyma that were significant enough to diagnose medical renal disease. Additionally, $69.3 \%$ of specimens had nonspecific changes that were categorized as 'concerning'(4). In light of such concerns, in 2009, the College of American Pathologists recommended pathologists closely examine the nonneoplastic renal parenchyma of nephrectomy specimens (5). Furthermore, it is now a clinical principle in the 2017 AUA guidelines to evaluate pathologic specimens for medical renal disease (2). Despite this, however, there is protocol about how these findings should be interpreted in the postoperative setting (5).

\section{Objectives}

We hypothesize that these non-neoplastic findings in the renal parenchyma correlate with increased risk of immediate post-operative changes in renal function. Additionally, we hypothesized that the rate of previously undiagnosed medical renal disease is underreported in existing literature.

\section{Patients and Methods \\ Patient selection}

We performed an Institutional Review Board (IRB) approved retrospective review of all patients that underwent partial nephrectomy, $\mathrm{RN}$, and nephroureterectomy at our institution by four urologic oncology surgeons from December 2009 to November 2013. Patients were excluded if they had a prior contralateral nephrectomy, currently on hemodialysis, prior solitary kidney, or underwent bilateral nephrectomy. Diagnosis of renal mass was typically based on radiologic findings with the exception of renal pelvic/ureteral lesions, which were confirmed with urine cytology and/or endoscopic biopsies. As is routine, renal biopsy is not required pre-operatively for diagnosis prior to intervention. The decision to perform a partial nephrectomy versus $\mathrm{RN}$ was at the discretion of the surgeon and was based on size, RENAL Nephrometry Score, and patient risk factors (6). Around 225 patients had complete pathologic and perioperative data for analysis. Patient parameters included age, race, gender, body mass index (BMI), preoperative and postoperative serum creatinine, preoperative hematuria, preoperative proteinuria, history of diabetes, and history of hypertension.

\section{Pathology data}

Pathology information collected regarding the neoplastic tissue included tumor size, tumor margin positivity, tumor type, tumor Fuhrman grade (if applicable), and tumor stage. Non-neoplastic parenchymal pathologic findings were categorized into interstitial abnormalities, glomerular abnormalities, and vascular abnormalities. Interstitial abnormalities included fibrosis, chronic inflammation, calcifications, tubular atrophy and thyroidization. Glomerular abnormalities included sclerosis, atrophy, and diabetic nephropathy. Vascular abnormalities included arteriosclerosis and hypertensive vasculitis.

\section{Ethical issues}

Human rights were respected in accordance with the Helsinki Declaration 1975, as revised in 1983. The ethical committee of MedStar Institutional Review Board (Code; 2014-161) approved the study. The informed consent was waived based on the nature of the protocol in accordance with IRB policy.

\section{Statistical analysis}

Data analysis was performed by the Department of Biostatistics and Bioinformatics at the MedStar Health Research Institute. A multivariate analysis was performed comparing patient information and pathologic findings with preoperative and postoperative renal function. Statistical significance was concluded with $P$ value of $\leq 0.05$. Logistic regression adjustments were performed to look at the effect of preoperative diabetes and CKD to see if they could be confounding factors.

\section{Results}

We reviewed the complete pathologic and perioperative data of 225 patients who underwent partial nephrectomy, $\mathrm{RN}$, and nephroureterectomy at our institution by four urologic oncology surgeons from December 2009 to November 2013. Of these, 103 underwent radical nephrectomies, 100 underwent partial nephrectomies and 22 underwent nephroureterectomies. The median age at time of surgery was 60.7 years (23-85). Around 53\% of patients were male and $47 \%$ female. Patient demographics are listed in Table 1.

Non-neoplastic pathologic abnormalities were found in $49 \%$ of specimens. The most common abnormalities were interstitial (ie, chronic inflammation and interstitial fibrosis). The prevalence of pathologic abnormalities is listed in Table 2.

Multivariate analysis was used to compare preoperative, immediate postoperative, and follow-up creatinine levels at follow up with the presence of non-neoplastic renal parenchyma abnormalities. Average time of follow up was 8 months. The presence of pathologic abnormality was significantly associated with increased serum creatinine levels postoperatively $(P=0.01)$ and at last follow up visit $(P=0.04)$. Univariate analysis found that glomerular and vascular abnormalities were each significantly associated with worsening renal function postoperatively and at 
Table 1. Patient and tumor characteristics

\begin{tabular}{|c|c|c|c|c|}
\hline & All Patients & Radical Nephrectomy & Partial Nephrectomy & Nephroureterectomy \\
\hline Total patients (n) & 225 & 103 & 100 & 22 \\
\hline Age (average) & 50.7 & 62.5 & 59.8 & 59.5 \\
\hline \multicolumn{5}{|l|}{ Gender } \\
\hline Male & 120 & 56 & 47 & 17 \\
\hline Female & 105 & 47 & 63 & 5 \\
\hline Tumor size $(\mathrm{cm})$, average & $5.2(0.6-19)$ & $7.1(1-19)$ & $3.1(0.5-7.3)$ & $5.4(2-15)$ \\
\hline \multicolumn{5}{|l|}{ Tumor type } \\
\hline Clear cell RCC & 102 & 53 & 47 & 2 \\
\hline Papillary RCC & 51 & 25 & 25 & 1 \\
\hline Urothelial cell & 21 & 2 & 0 & 18 \\
\hline AML & 10 & 6 & 6 & 0 \\
\hline Oncocytoma & 11 & 4 & 7 & 0 \\
\hline Chromophobe & 23 & 11 & 12 & 0 \\
\hline Granular & 2 & 1 & 1 & 0 \\
\hline Other & 4 & 2 & 2 & 0 \\
\hline Benign (no abnormalities) & 1 & 0 & 1 & 0 \\
\hline
\end{tabular}

Abbreviations: AML, acute myeloid leukemia; RCC, renal cell carcinoma.

follow-up. Interstitial abnormalities were not associated with worsening renal function (Table 3 ).

Further investigation of the sub-groups found no significant associations between pathologic abnormalities and renal dysfunction in the $\mathrm{RN}$ or the nephroureterectomy populations. However, there was a significant correlation between having any pathologic abnormalities and elevated

Table 2. Prevalence of non-neoplastic pathologic abnormalities

\begin{tabular}{lcc}
\hline & Present & Absent \\
\hline Any pathology abnormality & $111(49 \%)$ & $115(51 \%)$ \\
Interstitial (Any) & $98(42 \%)$ & $138(68 \%)$ \\
Chronic inflammation & $62(36 \%)$ & $144(64 \%)$ \\
Interstitial fibrosis & $57(25 \%)$ & $169(75 \%)$ \\
Calcifications & $24(11 \%)$ & $202(89 \%)$ \\
Thyroidization & $7(3 \%)$ & $219(97 \%)$ \\
Tubular Atrophy & $4(2 \%)$ & $222(98 \%)$ \\
Glomerular (Any) & $40(22 \%)$ & $176(78 \%)$ \\
Glomerular sclerosis & $38(17 \%)$ & $188(83 \%)$ \\
Glomerular atrophy & $8(4 \%)$ & $218(96 \%)$ \\
Vascular (Any) & $42(19 \%)$ & $154(81 \%)$ \\
Arteriosclerosis & $36(16 \%)$ & $190(84 \%)$ \\
Vascular hypertension & $5(3 \%)$ & $220(97 \%)$ \\
\hline
\end{tabular}

serum creatinine levels at follow up $(P=0.03)$.

Discussion

Chronic kidney disease has been found to put patients at increased risk of death, with the absolute risk of death increasing exponentially with decreasing renal function (7). In particular, these patients tend to be at increased risk of cardiovascular deaths. Thus, the importance of identifying patients at risk of postoperative CKD cannot be understated. Avoidance of renal injury led to the adoption of nephron- sparing surgical techniques to maximize preservation of renal parenchyma compared to $\mathrm{RN}$. Several retrospective studies have shown the benefits in renal function in partial nephrectomy patients (7-9).

Yet, without long-term follow up, the survival benefit of avoiding CKD may have been assumed rather than proven. In one of the only randomized prospective multicenter studies available on the subject, Van Poppel et al in 2011 found no difference in overall survival for patients undergoing radical versus partial nephrectomies with a median follow-up of 9.3 years (10). In fact, they found an overall increase in cardiovascular deaths in the NSS population compared with the $\mathrm{RN}$ population. Their overall conclusions were that NSS and RN both provide good oncologic outcomes with no significant differences

Table 3. Pre- and post-operative serum creatinine levels and renal pathology

\begin{tabular}{lccccccccccccc}
\hline & \multicolumn{3}{c}{ Interstitial abnormalities } & \multicolumn{2}{c}{ Glomerular abnormalities } & \multicolumn{3}{c}{ Vascular abnormalities } & \multicolumn{2}{c}{ Any pathologic abnormality } \\
\cline { 2 - 12 } & Present & Absent & $\boldsymbol{P}$ & Present & Absent & $\boldsymbol{P}$ & Present & Absent & $\boldsymbol{P}$ & Present & Absent & $\boldsymbol{P}$ \\
\hline Preoperative $\mathrm{Cr}$ & $1.3(1.4)$ & $1.2(1.4)$ & 0.17 & $1.4(1.8)$ & $1.2(1.2)$ & 0.48 & $1.5(2.0)$ & $1.2(1.2)$ & 0.17 & $1.3(1.3)$ & $1.2(1.4)$ & 0.06 \\
Postoperative $\mathrm{Cr}$ & $1.6(1.3)$ & $1.4(0.9)$ & 0.4 & $1.9(1.5)$ & $1.4(0.9)$ & $\mathbf{0 . 0 3}$ & $1.8(1.5)$ & $1.4(1.0)$ & $\mathbf{0 . 0 0 1}$ & $1.7(1.2)$ & $1.3(0.9)$ & $\mathbf{0 . 0 1}$ \\
Last follow up Cr & $1.5(1.1)$ & $1.4(1.3)$ & 0.3 & $1.7(0.8)$ & $1.4(1.3)$ & $\mathbf{0 . 0 4}$ & $1.7(0.8)$ & $1.4(1.3)$ & $\mathbf{0 . 0 4}$ & $1.6(1.0)$ & $1.4(1.3)$ & $\mathbf{0 . 0 4}$ \\
\hline
\end{tabular}


in overall survival. Despite the conclusions of Van Poppel et al, the general trend has been moving toward nephron sparing surgical options. In fact, in 2009, the AUA recommended partial nephrectomy for T1a renal masses as the standard of care for lesions in technically feasible locations with the intent of preserving renal function while maintaining similar oncologic outcomes to RN (11).

Because of these controversies, researchers have looked extensively at surgical techniques (minimizing clamp times and enucleation) to improve on the outcomes of NSS so that overall morbidity and mortality risks are reduced. Our finding that glomerular and vascular abnormalities correlate to increased risk of renal dysfunction suggests another important factor in non-neoplastic outcomes.

In 2009, the College of American Pathologists recommended pathologists institute to closer examination of the non-neoplastic renal parenchyma of nephrectomy specimens. However, there was no protocol about how these findings should be interpreted in the postoperative setting. Previously, Garcia-Roig et al retrospectively reviewed data of 45 patients who underwent partial nephrectomy and found that interstitial fibrosis was the only pathologic finding that was associated with preoperative serum creatinine (12). Our experience did not correlate. Salvatore et al reviewed pathologic findings in 381 patients who underwent partial and radical nephrectomies and found that postoperative creatinine levels were elevated in patients with severe arteriosclerosis, glomerulosclerosis, and tubular atrophy. Similar to Salvatore et al, we found glomerular and vascular abnormalities being associated with postoperative increases in serum creatinine in our univariate analysis (4). It remains unclear why glomerular and vascular pathologies had a stronger tendency towards renal dysfunction versus interstitial changes.

When we compared the outcomes of our patients based on the type of surgery they underwent, we only found a correlation between elevated postoperative creatinine levels (and GFR), and the existence of any pathologic abnormalities in the patients who underwent partial nephrectomy. It is difficult to say whether the lack of such a correlation within the RN or nephroureterectomy populations was because our study was underpowered. On the other hand, differences in postoperative serum creatinine secondary to underlying medical renal disease may be more exaggerated in patients undergoing partial nephrectomy secondary to surgical technique including warm ischemia. Further studies with larger patient populations and longer follow-up will hopefully elucidate the reasoning for these findings. None of our patients went on to severe renal dysfunction requiring dialysis in the immediate post-operative period.

An additional consideration from our findings may be a stronger case for partial nephrectomy. When patients have undiagnosed abnormalities in their non-neoplastic renal parenchyma, it may be imperative for the surgeon to spare nephrons where oncologically possible. Furthermore, our series may add context to the case for pre- operative renal biopsy. Confirmation of malignancy when possible and sampling of non-neoplastic tissue before surgery may aid in both pre- and intra-operative decision making and patient counseling. There has been an increase in the use of multiple core renal tumor biopsies as it has been shown to increase the diagnostic rate (13). Further studies may help to elucidate such considerations.

Long-term follow up is lacking in retrospective studies regarding non-neoplastic renal pathology findings. This leads to limited prognostic benefits from our findings as well. Future studies with longer follow-ups would help to determine whether renal dysfunction in nephrectomy patients with parenchymal abnormalities continues to progress postoperatively and how quickly it worsens. Until then, based on our findings, we suggest that patients with pathology findings consistent with undiagnosed medical renal disease be considered for nephrology referral earlier in the postoperative setting to help prevent further renal injury and delay potential CKD.

\section{Conclusion}

Our research suggests that abnormalities in non-neoplastic renal parenchyma found in renal specimens after $\mathrm{RN}$ can show early manifestations in the acute setting with elevated serum creatinine. This information may even compel surgeons more strongly towards early nephrology referral and/or renal biopsy and partial nephrectomy. Post-operatively, these findings may help determine which patients should be followed more closely. These findings further support the guidelines published in 2017. Studies with longer follow up times would be beneficial in helping to determine if changes in postoperative management of these patients needs to be made.

\section{Limitations of the study}

As noted above our study is limited by retrospective design and relatively limited follow up. Additionally, our data now support established guidelines for the testing and referral of appropriate patients to nephrology if medical renal insufficiency is diagnosed. Finally, as our cohort is mixed, with radical, partial, and nephroureterectomy specimens, the applicability to any one population may be in question.

\section{Acknowledgements}

MedSTAR Health Research Institute; Washington Hospital Center Department of Pathology; Washington Hospital Center Department of Nephrology

\section{Authors' contribution}

$\mathrm{JK}, \mathrm{KV}$, and MV designed the study, observed accuracy and validity of the study. FH and LB collected the data and did much of the analysis. NS finalized analysis and refined 
data. MV supervised the project. NS and FH wrote the paper. All authors edited and revised the final manuscript and accepted its publication

\section{Conflicts of interest}

The authors declared no competing interests.

\section{Ethical considerations}

Ethical issues (including plagiarism, data fabrication, double publication) have been completely observed by the authors.

\section{Funding/Support}

No funding.

\section{References}

1. Howlader N NA, Krapcho M, Garshell J, Miller D, Altekruse SF, Kosary CL, et al. SEER Cancer Statistics Review, 19752011. 2014. [March 19, 2015]. http://seer.cancer.gov/ csr/1975_2011/

2. Campbell S, Uzzo RG, Allaf ME, Bass EB, Cadeddu JA, Chang A, et al. Renal Mass and Localized Renal Cancer: AUA Guideline. J Urol. 2017;198:520-529. doi: 10.1016/j. juro.2017.04.100.

3. Campbell SC, Novick AC, Belldegrun A, Blute ML, Chow GK, Derweesh IH, et al. Guideline for management of the clinical T1 renal mass. J Urol. 2009;182:1271-9. doi: 10.1016/j.juro.2009.07.004.

4. Salvatore SP, Cha EK, Rosoff JS, Seshan SV. Nonneoplastic renal cortical scarring at tumor nephrectomy predicts decline in kidney function. Arch Pathol Lab Med. 2013;137:531-40. doi: 10.5858/arpa.2012-0070-OA.

5. Srigley JR, Amin MB, Delahunt B, Campbell SC, Chang A, Grignon DJ, et al. Protocol for the examination of specimens from patients with invasive carcinoma of renal tubular origin. Arch Pathol Lab Med. 2010;134:e25-30. doi: 10.1043/1543-2165-134.4.e25.

6. Kutikov A, Uzzo RG. The R.E.N.A.L. nephrometry score: a comprehensive standardized system for quantitating renal tumor size, location and depth. J Urol. 2009;182:844-53. doi: 10.1016/j.juro.2009.05.035.

7. Tonelli M, Wiebe N, Culleton B, House A, Rabbat C, Fok $\mathrm{M}$, et al. Chronic kidney disease and mortality risk: a systematic review. J Am Soc Nephrol. 2006;17:2034-47. doi: 10.1681/ASN.2005101085.

8. Lane BR, Chen H, Morrow M, Anema JG, Kahnoski RJ. Increasing use of kidney sparing approaches for localized renal tumors in a community based health system: impact on renal functional outcomes. J Urol. 2011;186:1229-35. doi: $10.1016 /$ j.juro.2011.05.081.

9. Huang WC, Levey AS, Serio AM, Snyder M, Vickers AJ, Raj GV, et al. Chronic kidney disease after nephrectomy in patients with renal cortical tumours: a retrospective cohort study. Lancet Oncol. 2006;7:735-40. doi: 10.1016/S14702045(06)70803-8.

10. Van Poppel H, Da Pozzo L, Albrecht W, Matveev V, Bono A, Borkowski A, et al. A prospective, randomised EORTC intergroup phase 3 study comparing the oncologic outcome of elective nephron-sparing surgery and radical nephrectomy for low-stage renal cell carcinoma. Eur Urol. 2011;59:543-52. doi: 10.1016/j.eururo.2010.12.013.

11. AUA. AUA Guideline: Full kidney removal not necessary for all kidney tumors. Am Urol Assoc. 2009.

12. Garcia-Roig M, Gorin MA, Parra-Herran C, GarciaBuitrago M, Kava BR, Jorda M, et al. Pathologic evaluation of non-neoplastic renal parenchyma in partial nephrectomy specimens. World J Urol. 2013;31:835-9. doi: 10.1007/ s00345-011-0720-1.

13. Leão RR, Richard PO, Jewett MA. The role of biopsy for small renal masses. Int J Surg. 2016;36:513-517. doi: 10.1016/j.ijsu.2016.02.097.

Copyright (c) 2020 The Author(s); Published by Nickan Research Institute. This is an open-access article distributed under the terms of the Creative Commons Attribution License (http://creativecommons.org/licenses/by/4.0), which permits unrestricted use, distribution, and reproduction in any medium, provided the original work is properly cited. 